\title{
Multimessenger Astronomy with Neutrinos
}

\author{
Francisco Salesa Greus ${ }^{1,2, *(1)}$ and Agustín Sánchez Losa ${ }^{1}[0$ \\ 1 IFIC - Instituto de Física Corpuscular (CSIC - Universitat de València), c/ Catedrático José Beltrán 2, \\ E-46980 Paterna, Spain; Agustin.Sanchez@ific.uv.es \\ 2 Institute of Nuclear Physics, Polish Academy of Sciences, PL-31342 Kraków, Poland \\ * Correspondence: sagreus@ific.uv.es; Tel.: +34-963543538
}

check for

updates

Citation: Salesa Greus, F.; Sánchez Losa, A. Multimessenger Astronomy with Neutrinos. Universe 2021, 7, 397. https://doi.org/10.3390/

universe7110397

Academic Editor: Maria Vasileiou

Received: 30 September 2021

Accepted: 17 October 2021

Published: 21 October 2021

Publisher's Note: MDPI stays neutral with regard to jurisdictional claims in published maps and institutional affiliations.

Copyright: (c) 2021 by the authors. Licensee MDPI, Basel, Switzerland. This article is an open access article distributed under the terms and conditions of the Creative Commons Attribution (CC BY) license (https:// creativecommons.org/licenses/by/ $4.0 /)$.

\begin{abstract}
Multimessenger astronomy is arguably the branch of the astroparticle physics field that has seen the most significant developments in recent years. In this manuscript, we will review the state-of-the-art, the recent observations, and the prospects and challenges for the near future. We will give special emphasis to the observation carried out with neutrino telescopes.
\end{abstract}

Keywords: multimessenger astronomy; astroparticle physics; neutrinos

\section{Introduction}

Astronomical observations have been traditionally done with visible light. From the times of Galileo times until now we have been able to expand the observation range to all the electromagnetic spectrum, from radio to gamma rays. Apart from photons, the discovery of other particles has opened the possibility of using them as cosmic messengers to explore the Universe. For instance, cosmic rays (CRs) were first discovered in the early 1910s. We know that CRs are ionized nuclei of extraterrestrial origin, and that they are produced and accelerated in a broad energy range, reaching energies above $10^{20} \mathrm{eV}$. The spectrum of the CRs detected at Earth decreases with the energy, following approximately a power law $\mathrm{E}^{-n}$ with $\mathrm{n} \sim 3$. The Sun is the main source of $\mathrm{CRs}$ below $\mathrm{GeV}$ energies. $\mathrm{Up}$ to PeV energies, CR are believed to be dominated by Galactic sources, and then, at the highest energies, CRs are likely of extragalactic origin [1]. As CRs are charged, their directionality is lost due to the Galactic magnetic field. This is probably one of the main reasons why the origin of the most energetic CRs is still unknown. We have also detected neutrinos from extraterrestrial origin. First from the Sun [2], and then from a nearby supernova explosion in 1987 [3-5] which can be considered as the birth of neutrino astronomy. By that time, the first project to construct a neutrino telescope was already ongoing [6]. However, only after decades of research and development, neutrino astronomy had its turning point in 2014 with the discovery of a high-energy cosmic neutrino flux by the IceCube collaboration [7]. The most recent cosmic messengers discovered are gravitational waves (GWs). The path to the first GW detection was also not easy, and it took a century from their theoretical prediction to the first confirmation in 2015 by the LIGO and VIRGO collaborations [8].

Multimessenger astronomy originates as a consequence of astrophysical neutrino and GW detection techniques reaching maturity. Multimessenger astronomy is based on the observation of four cosmic messengers, namely, photons, CRs, neutrinos, and GWs. The detection in coincidence of all, or some, of these messengers allows the study of a source in a similar fashion as it has been done with multiwavelength electromagnetic observations. Moreover, the fact of involving different types of particles adds extra information coming from interactions involving all fundamental forces of nature.

We have divided this review into three sections. In Section 2, we will discuss the two events that marked the start of the multimessenger era, four years ago. In Section 3, we will review the most recent results and what we have learned from them. To conclude, in Section 4, we will discuss the future prospects and challenges in the field. 


\section{Multimessenger Astronomy Milestones}

Excluding the solar neutrino detection, the observation by chance of neutrinos coming from the supernova explosion SN1987A is the first astronomical event producing a multimessenger (photon-neutrino) coincidence. However, there are two main events, both happening in 2017, that really marked the birth of a new field in astrophysics, multimessenger astronomy.

The first event was the result of two neutron stars merging into a black hole. This produced a GW (GW170817) that was detected by the LIGO and Virgo [9] Scientific Collaborations. Less than 2 seconds after the event, a short gamma-ray burst (GRB) (GRB 170817A) was detected by the Fermi and INTEGRAL satellites. This coincidence triggered a campaign where several observatories followed-up the event in an unprecedented way [10]. Thanks to this coincident detection it was possible to determine the location and the type of sources involved, bringing the first experimental evidence of a kilonova [11], a type of transient event, theoretically predicted more than two decades ago [12], where nucleosynthesis of the heavy elements is produced.

The second event (IC-170922A) was triggered by a high-energy neutrino, of about $300 \mathrm{TeV}$, detected by the IceCube observatory on 22 September 2017. Again, this event was extensively followed up by other observatories. In this case, observations from the Fermi-LAT satellite were able to point out a blazar (TXS0506+056) in active state which was in spatial and temporal coincidence with the neutrino event. The event was rejected to be produced by background fluctuations at $3 \sigma$ level [13]. After this detection, archival analysis of IceCube data prior to the IceCube-170922A event unveiled a potential flare in neutrinos [14], between September 2014 and March 2015, with 3.5 $\sigma$ statistical significance and independent of the 2017 neutrino alert. In this case, no gamma-ray counterpart was observed. An extensive multiwavelength monitoring of TXS0506+056 started after the coincident event in September 2017 showed a low state emission except for December 1st and 3rd, 2018 with a flare comparable to the one in 2017 [15]. However, no neutrino excess was observed. It is also important to mention that TXS0506+056 came out as the second most significant source ( $2.8 \sigma$ pre-trial) in the point source search analysis done with the ANTARES neutrino telescope using a pre-selected list of sources [16], which makes the case of TXS0506+056 stronger.

\section{Recent Results}

Once it has been well established that a flux of high-energy neutrinos of cosmic origin exist [7], the next step is to disentangle it and identify which are the sources. The most recent all-sky searches performed by ANTARES [16] and IceCube [17] did not reveal any significant detection above the discovery threshold of $5 \sigma$, with the excess near the galaxy NGC 1068 observed by IceCube being the most interesting spot with a post-trial significance of 2.9 $\sigma$. This type of high-energy searches benefit from multimessenger astronomy thanks to including the sky coordinates and timing information from potential cosmic messenger counterparts. That was how the first evidence of a cosmic neutrino source, TXS0506+056, was found.

Understanding the multimessenger emission from TXS0506+056 has been challenging from the theoretical point of view, as it is difficult to get a good agreement between the observed neutrino signal and other wavelength observations. If one tries to explain it with a single-zone model, i.e., both gammas and neutrinos coming from the same region, one finds out that a leptonic scenario with a radiatively subdominant hadronic component provides the only physically consistent single-zone picture [18]. A higher neutrino flux would be expected if the source hosts two physically distinct emitting regions (see for instance [19]). However, current observations cannot discriminate between single- or multizone emission models. Related to this, a compelling neutrino-radio correlation [20] has been recently discovered. The authors proposed that neutrinos and gamma rays may be indeed produced in different regions [21]. If this is actually the case, X-ray and radio may be better wavelengths when looking for photon-neutrino correlations. The correlation with 
radio blazars is also supported by ANTARES observations [22]. In this regard, ANTARES has recently reported the results from an untriggered search from radio blazars with an interesting association coming from J0242+1101 [23].

Thanks to the IceCube alert system [24], which is presently providing on the order of 10 (20) gold (bronze) alerts per year ${ }^{1}$, more coincidences between neutrino and blazars have been found lately. For instance, PKS 1502+106 blazar was coincident with a $300 \mathrm{TeV}$ neutrino [25]. In this case, the blazar was in a quiescent state at the time of the neutrino alert. However, no more neutrinos were detected. Another example is 3HSP J095507.9, which was also coincident with a high-energy neutrino detected by IceCube [26,27]. However, for this event a lot of sources lay around the best position provided by IceCube, preventing the identification of a potential source candidate. This also underscores that sub-degree angular resolution, achievable by future neutrino observatories, will be key when looking for spatial coincidences. From Fermi-LAT observations we know that blazars are the most abundant extragalactic gamma-ray sources, constituting roughly $80 \%$ of the entire extragalactic source population [28]. However, current predictions based on stacking catalog searches performed with IceCube data estimate that neutrinos emitted by blazars, in the range between around $10 \mathrm{TeV}$ and $2 \mathrm{PeV}$, can only contribute up to $27 \%$ to the total neutrino diffuse flux [29]. More multimessenger observations with next generation experiments are required to test current theoretical models, and therefore provide valuable information to understand the particle production and acceleration in blazars.

Apart from blazars other neutrino candidates have been already identified thanks to multimessenger observations. This is the case of Tidal Disruption Events (TDEs), which are the result of a star being ripped apart when passing next to a supermassive black hole. TDEs were already hypothesized as possible neutrino sources, e.g., in [30]. However, it was not until 2019 when an IceCube neutrino, with 59\% probability of being of astrophysical origin (IC-191001A), triggered an alert that was followed-up by the Zwicky Transient Facility [31]. In spatial coincidence with the neutrino alert a TDE (AT2019dsg) was observed. Given that TDEs are rare events, the chance probability of finding this coincident event was estimated to be less than $0.5 \%$ [32]. After the first neutrino-TDE coincidence, more recently another possible association has been detected (IC200530A with AT2019fdr) which has been considered by some scientists as evidence of an emerging trend. The chance probability of finding this second event in coincidence was also small. Both events have been followed up by ANTARES, however did not produce any significant neutrino excess [33]. The non detection does not contradict the observation by IceCube, as the sensitivity of ANTARES was above the neutrino flux prediction. On the other hand, there are preliminary indications of an excess in GVD-Baikal data [34]. Current estimations predict that TDEs contribute at least $2 \%$ but not more than $40 \%$ of the total neutrino flux [35]. Again, more observations are needed to confirm TDEs as sources of high-energy neutrinos and determine their precise contribution to the diffuse neutrino flux.

GRBs are another type of sources that have long been predicted as good candidates to emit high-energy neutrinos, see, for instance, in [36]. However, so far, all the searches have been unsuccessful $[37,38]$. Recently, some studies have tried to infer what would be the relative contribution of the different neutrino candidate sources, see, e.g., in [39]. However, it seems that there is no clear indication of a dominant type of source producing cosmic neutrinos. Interestingly enough, the same study suggest that there is room for unknown sources.

\section{Future Prospects and Challenges}

\subsection{Future Instruments and Instrument Upgrades}

Regarding neutrino telescopes there are three major projects that will be operating in the near future. KM3NeT [40] is a research infrastructure being built in the Mediterranean sea. KM3NeT is composed of two detectors, first ARCA (Astroparticle Research with Cosmics in the Abyss) which is designed to be sensitive to high-energy neutrinos in the TeV$\mathrm{PeV}$ range, and therefore with astrophysics studies as the main goal. The second detector 
is called ORCA (Oscillation Research with Cosmics in the Abyss) and it is sensitive to GeV neutrinos. The ORCA detector will primarily be used for the study of neutrino properties. Both instruments will use the same technology and detection principle, i.e., array of photomultipliers tubes (PMTs) in sea water, being the main difference the volume covered, and therefore the PMT density. ARCA is expected to be fully operational in 2027 and ORCA in 2025.

Moreover, in water there is the GVD-Baikal project that is in construction in the Baikal Lake in Russia. GVD-Baikal is currently operational with 2304 optical modules arranged in eight clusters of eight strings each. In the present configuration, it has an effective volume of $0.4 \mathrm{~km}^{3}$ for cascades with energy above $100 \mathrm{TeV}$ [41]. Current plans are to deploy six additional clusters for the period from 2022 to 2024 which should provide an additional $0.3 \mathrm{~km}^{3}$ effective volume.

The leading project in neutrino telescopes in the last decade has been IceCube [42] which is a neutrino telescope installed in the South Pole. After 10 years of successful operation there are plans for two major upgrades. One, called IceCube-Gen2 [43], expected to be completed by the early 2030s, will significantly increase the IceCube effective volume and energy range sensitivity. The other, called IceCube-Upgrade [44], represents a fraction of a larger project called Precision IceCube Next Generation Upgrade (PINGU) [45], which aims to increase the sensitivity to lower energies even more than with IceCube-DeepCore, mainly thanks to a higher string density, and that will mostly study neutrino properties.

We can also add to the list of future intended neutrino telescopes the Pacific Ocean Neutrino Experiment (P-ONE) [46]. The P-ONE project is presently in research and development phase. The goal of the collaboration is to install a multi-cubic-kilometer neutrino telescope in the Pacific Ocean, which is expected to be operational in the next decade.

Other neutrino experiments, foreseen to be operational by the end of this decade, like Hyper-Kamiokande [47] and DUNE [48], will be sensitive to lower neutrino energies (MeV$\mathrm{GeV})$. However, they can still contribute to get the whole picture of some astrophysical events. For instance, the explosion of a nearby supernova, where low-energy neutrinos are expected to be produced.

There are also very exciting plans for next generation experiments aiming to detect other cosmic messengers. Just to mention a few of them, we have the Cherenkov Telescope Array (CTA) [49] with two planned sites (Northern and Southern Hemisphere), and LHAASO [50], fully operational since July 2021, detecting gamma rays. KAGRA [51], detecting GWs, will join LIGO and Virgo for the next GW data taking run (O4), which is expected to start in late 2022. Finally, detecting ultra-high-energy CRs, there will be AugerPrime [52], the upgrade of the Pierre Auger Observatory. Such a network of observatories, distributed in different locations around the globe (see Figure 1), will provide a full multimessenger coverage of the sky.

\subsection{Alert Systems and Strategies}

Considering the number of experiments currently being under construction and planned, it seems clear that an efficient communication between collaborations is crucial. Moreover, for the case of pointing instruments, like CTA, this communication also needs to be fast. Neutrinos are actually very good messengers to trigger alerts because they are able to easily escape from sources. These neutrino alerts can give an early warning to other observatories of an incoming event, allowing for a prompt follow up of transient phenomena.

There are already ways to announce, in real-time, interesting events to the astrophysics community, e.g., the GammaRay Coordinates Network (GCN) [53]. In addition to these announcements, there are also sites, like the Astronomers Telegram (ATEL) [54], where a brief report about recent observations made by the experiments is posted online. Still in beta testing phase there is a project called Astro-COLIBRI [55] whose goal is to act as a central platform where a large set of information coming from different experiments is gathered. This can be accessed via web or smartphone interface. The data will be immediately available and will contain relevant information such as the visibility of the 
event for a given observatory, the false alarm rate, or the probability of the event to be of astrophysical origin.

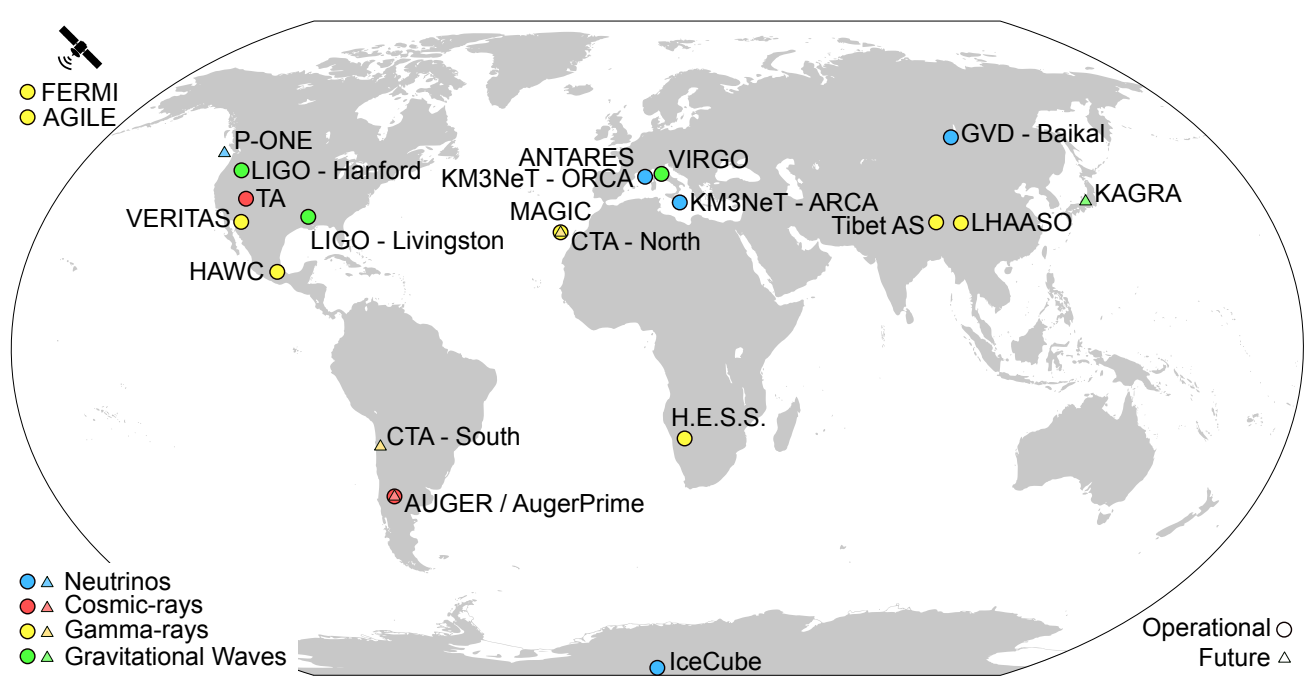

Figure 1. Earth map indicating the location of a selection of multimessenger observatories that are either currently operating (circles) or planned (triangles). Satellites are depicted outside to the map.

In addition to these prompt alert systems across collaborations, there are alert followup programs like TaToO [56], where the most promising ANTARES events trigger a prompt optical, radio, and X-ray follow-up on the sky region where the neutrino candidate comes from, looking for any potential transient counterpart within hours, days, and months since the event. This is done using a network of optical telescopes at different locations (at a rate of 25 alerts per year) and, for the most energetic ones (around 6 alerts per year), the XRT instrument aboard the Swift satellite and the Murchison Wide field Array radio telescope [57].

As another example of how data from different experiments is shared, we have the Astrophysical Multimessenger Observatory Network (AMON) [58]. One of the main ideas of AMON is to use sub-threshold data from different experiments to exploit the fact that a combined detection is expected to increase the significance of the event. Therefore, an event that by itself is not enough to claim a detection with a single experiment, and could have been rejected, can actually become significant when detected in coincidence with other observatories. An example of a recent analysis done through this network is [59], focused on gamma-ray and neutrino coincidences.

\subsection{Future Challenges}

Concerning the multimessenger astronomy goals in the next few years, one thing that should be attainable very soon is a firm confirmation of a source of cosmic neutrinos above the discovery threshold of $5 \sigma$. To this end, the selection of the most promising sources, to reduce the amount of trials in the search, thanks to multimessenger observations will be crucial. This is quite likely to be accomplished by more than one project which will provide an unbiased way of measuring the spectrum of the sources, bringing key information to understand the high-energy neutrino production and acceleration mechanisms in the source. Also combined analyses are possible, as has been already done in the past [60].

One multimessenger observation that is most awaited, and can probably be achieved thanks to the improved sensitivity of the future experiments, is the coincident detection of a GW event with high-energy neutrinos. Thanks to recent GW observations, we have a better understanding of the link between neutron star mergers and short GRBs, and the physics involved. We already discussed the particular case of the binary neutron star merger GW170817, which led to the GRB170817A coincidence. This type of event should produce a GW signature together with gamma rays and neutrinos. However, at present, the only 
confirmed coincident detection is the GW-gamma correlation, while no evidence of neutrino emission was found [61,62]. The theoretical estimations of neutrino production [63] from GW170817 show that the expected flux should be already detectable, with current neutrino telescopes, under favorable circumstances, see Figure 2.

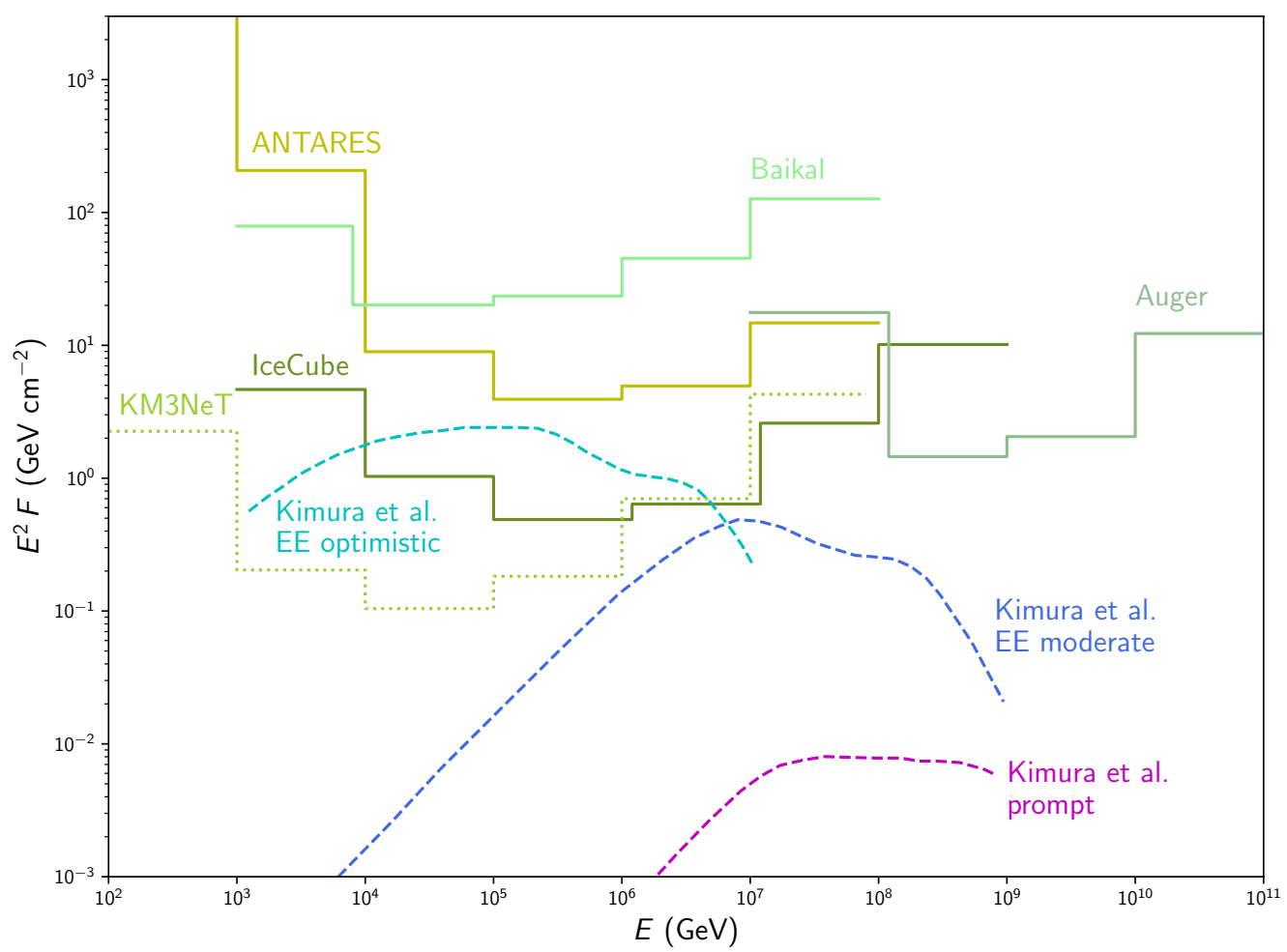

Figure 2. Fluence upper limits, per flavor, on the high-energy neutrino emission from experimental data assuming a \pm 500 s time window around the GW170817 event [61]. Baikal limits are from [62]. KM3NeT preliminary sensitivity, computed for an optimal zenith angle, is from [64]. Theoretical models for comparison are from [63]. Figure adapted from the work in [61].

Apart from the detection of high-energy neutrinos, the detection of the prompt emission in gamma-rays by observatories like HAWC or LHAASO will be essential to understand how these energetic explosions work.

Another open question to be addressed by multimessenger observations is the connection between ultra-high-energy CRs, gamma-rays, and high-energy neutrinos. Intensity of gamma-rays, neutrinos, and UHECRs has been shown to be comparable (see Figure 3), suggesting that they may be powered by the same sources. As blazars are the most abundant extragalactic sources, they are by default the most promising candidates. However, blazars do not seem to fit the bill, as they are subdominant in the high-energy neutrino flux [65]. 


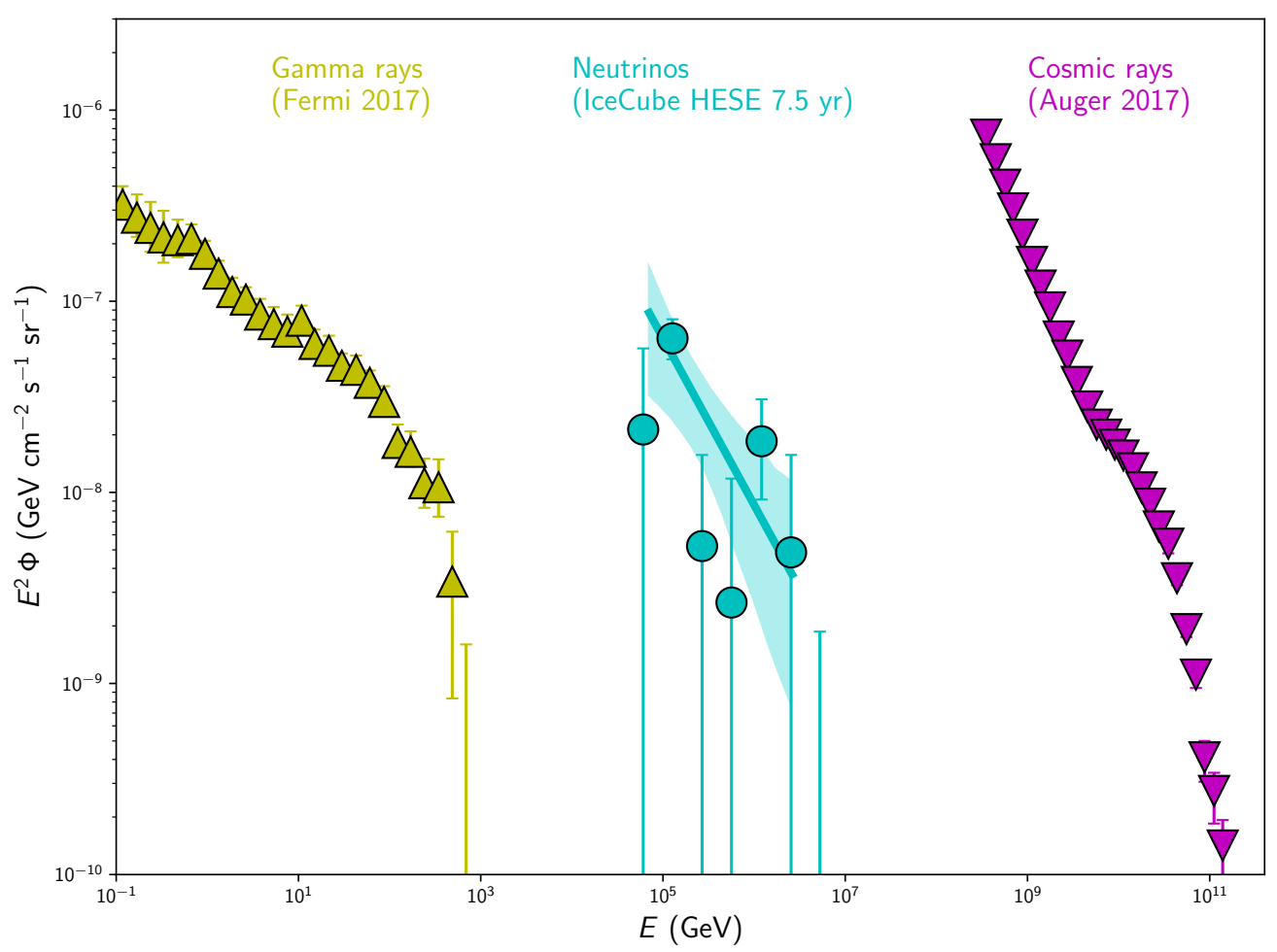

Figure 3. High-energy fluxes of gamma rays [66], neutrinos [67], and cosmic rays [68]. Figure adapted from the work in [67].

Apart from the usual suspects (e.g., GRBs and TDEs), the case for star-forming galaxies as common sources has recently grown in popularity thanks to the work in [69] where the authors claim that the diffuse gamma-ray flux detected by Fermi-LAT [66] is actually dominated by star-forming galaxies. At the same time, the data collected in the Pierre Auger Observatory showed an indication of anisotropy at $4.0 \sigma$ level in the arrival direction of CRs with $\mathrm{E}>39 \mathrm{EeV}$ showing an excess from the direction of nearby starburst galaxies [70]. Claiming that this type of high-rate star-forming galaxies can be the cause of $\sim 10 \%$ of the ultra-high-energy CR flux. It has been also shown that the contribution of the starburst galaxies to the neutrino diffuse flux is sub-dominant and constrained to be at the level of $\sim 10 \%$ [71]. Therefore, it remains unanswered for now whether there is a dominant type of source accelerating all cosmic messengers.

Finally, if we restrict the search to our Galaxy, one of the questions that will be solved in the near future thanks to multimessenger observations is what are the Galactic $\mathrm{CR}$ sources and how those CR propagate through the Galaxy. Regarding this, it has been shown that a sub-PeV diffuse Galactic gamma-ray emission exists [72]. Based on this result in [73], the authors showed that the Galactic neutrino contribution should constitute roughly $5-10 \%$ of the IceCube diffuse flux and that, in the $10-100 \mathrm{TeV}$ range, the expected Galactic neutrino flux should be comparable to the total neutrino diffuse flux. If so, the next-generation neutrino telescopes should be sensitive enough to detect it. It is possible that part of the measured gamma-ray diffuse flux comes from individual sources, being the obvious candidates the very-high-energy sources detected by HAWC [74] and LHAASO [75]. Combined multimessenger observations should be able to confirm whether this is actually the case. In fact, there have been claims for evidence for such a joint production, of high-energy neutrinos and gamma rays, in the Cygnus Coocon region, based on the correlation of a high-energy IceCube neutrino and a high-energy $(>300 \mathrm{TeV})$ photon flare observed by the Carpet-2 experiment [76]. 


\section{Outlook}

Multimessenger astronomy is a new branch of astroparticle physics, for which neutrinos are expected to play a key role. It took just a few events, detected in coincidence, to demonstrate that combining different messengers has a great potential for discoveries.

Questions like what is the origin of the ultra-high energy cosmic rays are likely to be answered thanks to multimessenger observations.

Considering the numerous facilities planned for the near future, or already taking data (KM3NeT, IceCube-Gen2, GVD-Baikal, P-ONE, CTA, LHAASO, KAGRA, AugerPrime, etc.), multimessenger astronomy has a bright future and is expected to revolutionize our understanding of the Universe in the next decade.

Author Contributions: All authors contributed equally to this article. All authors have read and agreed to the published version of the manuscript.

Funding: This work was funded by Generalitat Valenciana, Spain (CIDEGENT/2018/034 and CIDEGENT / 2020/049 grants).

Institutional Review Board Statement: Not applicable.

Informed Consent Statement: Not applicable.

Data Availability Statement: Not applicable.

Acknowledgments: The authors thank the Valencia Experimental Group on Astroparticle Physics (VEGA) and the Ministerio de Ciencia, Innovación, Investigación y Universidades (MCIU): Programa Estatal de Generación de Conocimiento (ref. PGC2018-096663-B-C41) (MCIU/FEDER).

Conflicts of Interest: The authors declare no conflict of interest.

\begin{tabular}{|c|c|}
\hline \multicolumn{2}{|c|}{ Abbreviations } \\
\hline & llowing abbreviations ar \\
\hline GW & Gravitational Wave \\
\hline CR & Cosmic Ray \\
\hline GRB & Gamma-Ray Burst \\
\hline TDE & Tidal Disruption Event \\
\hline PMT & Photomultipliers tubes \\
\hline
\end{tabular}

Note

1 Gold (bronze) alerts are neutrino events with $>50 \%(>30 \%)$ probability of being from astrophysical origin.

\section{References}

1. Zyla, P.A. et al. [Particle Data Group]. Review of Particle Physics. Prog. Theor. Exp. Phys. 2020, 8, 083 C01.

2. Davis, R., Jr.; Harmer, D.S.; Hoffman, K.C. Search for neutrinos from the sun. Phys. Rev. Lett. 1968, 20, 1205. [CrossRef]

3. Hirata, K.S.; Kajita, T.; Koshiba, M.; Nakahata, M.; Oyama, Y.; Sato, N.; Suzuki, A.; Takita, M.; Totsuka, Y.; Kifune, T.; et al. Observation in the Kamiokande-II Detector of the Neutrino Burst from Supernova SN 1987a. Phys. Rev. D 1988, 38, 448. [CrossRef]

4. Haines, T. et al. [IMB Collaboration]. Neutrinos from SN1987a in the IMB detector. Nucl. Instrum. Meth. A 1988, 264, 28-31. [CrossRef]

5. Alekseev, E.; Alexeyeva, L.N.; Krivosheina, I.V.; Volchenko, V.I. Detection of the neutrino signal from SN 1987A in the LMC using the INR Baksan underground scintillation telescope. Phys. Lett. B 1988, 205, 209-214. [CrossRef]

6. Babson, J. et al. [DUMAND Collaboration]. Cosmic Ray Muons in the Deep Ocean. Phys. Rev. D 1990, 42, 3613. [CrossRef] [PubMed]

7. Aartsen, M.G. et al. [IceCube Collaboration]. Observation of High-Energy Astrophysical Neutrinos in Three Years of IceCube Data. Phys. Rev. Lett. 2014, 113, 101101. [CrossRef] [PubMed]

8. Abbott, B.P. et al. [LIGO Scientific Collaboration and Virgo Collaboration]. Observation of Gravitational Waves from a Binary Black Hole Merger. Phys. Rev. Lett. 2016, 116, 061102. [CrossRef] [PubMed]

9. Abbott, B.P. et al. LIGO Scientific Collaboration and Virgo Collaboration]. GW170817: Observation of Gravitational Waves from a Binary Neutron Star Inspiral. Phys. Rev. Lett. 2017, 119, 161101. [CrossRef] 
10. Abbott, B.P. et al. [AST3, CAASTRO, VINROUGE, MASTER, J-GEM, GROWTH, JAGWAR, CaltechNRAO, TTU-NRAO, NuSTAR, Pan-STARRS, MAXI Team, TZAC Consortium, KU, Nordic Optical Telescope, ePESSTO, GROND, Texas Tech University, SALT Group, TOROS, BOOTES, MWA, CALET, IKI-GW Follow-up, H.E.S.S., LOFAR, LWA, HAWC, Pierre Auger, ALMA, Euro VLBI Team, Pi of Sky, Chandra Team at McGill University, DFN, ATLAS Telescopes, High Time Resolution Universe Survey, RIMAS, RATIR, SKA South Africa/MeerKAT]. Multi-messenger Observations of a Binary Neutron Star Merger. Astrophys. J. Lett. 2017, 848, L12. [CrossRef]

11. Metzger, B.D. Kilonovae. Living Rev. Relativ. 2017, 20, 3. [CrossRef]

12. Li-Xin, L.; Paczyński, B. Transient Events from Neutron Star Mergers. Astrophys. J. 1998, 507, L59-L62. [CrossRef]

13. Aartsen, M.G. et al. [IceCube Collaboration]. Multimessenger observations of a flaring blazar coincident with high-energy neutrino IceCube-170922A. Science 2018, 361, eaat1378. [CrossRef] [PubMed]

14. Aartsen, M.G. et al. [IceCube Collaboration]. Neutrino emission from the direction of the blazar TXS $0506+056$ prior to the IceCube-170922A alert. Science 2018, 361, 147-151. [CrossRef] [PubMed]

15. Satalecka, K. et al. [MAGIC, ATCA, OVRO and TELAMON Collaborations]. Multi-epoch monitoring of TXS $0506+056$ with MAGIC and MWL partners. In Proceedings of the International Cosmic Ray Conference (ICRC2021), Berlin, Germany, 12-23 July 2021; Volume 395, p. 875.

16. Illuminati, G. et al. [ANTARES Collaboration]. Searches for point-like sources of cosmic neutrinos with 13 years of ANTARES data. In Proceedings of the International Cosmic Ray Conference (ICRC2021), Berlin, Germany, 12-23 July 2021; Volume 395, p. 1161.

17. Aartsen, M.G.; Ackermann, M.; Adams, J.; Aguilar, J.A.; Ahlers, M.; Ahrens, M.; Alispach, C.; Andeen, K.; Anderson, T.; Ansseau, I.; et al. Time-Integrated Neutrino Source Searches with 10 Years of IceCube Data. Phys. Rev. Lett. 2020, 124, 05110. [CrossRef]

18. Keivani, A.; Murase, K.; Petropoulou, M.; Fox, D.B.; Cenko, S.B.; Chaty, S.; Coleiro, A.; DeLaunay, J.J.; Dimitrakoudis, S.; Evans, P.A.; et al. A Multimessenger Picture of the Flaring Blazar TXS 0506+056: Implications for High-Energy Neutrino Emission and Cosmic Ray Acceleration. Astrophys. J. 2018, 864, 84. [CrossRef]

19. Xue, R.; Liu, R.Y.; Petropoulou, M.; Oikonomou, F.; Wang, Z.R.; Wang, K.; Wang, X.Y. A Two-zone Model for Blazar Emission: Implications for TXS 0506+056 and the Neutrino Event IceCube-170922A. Astrophys. J. 2019, 886, 23, doi: 10.3847/1538-4357/ab4b44 [CrossRef]

20. Plavin, A.; Kovalev, Y.Y.; Kovalev, Y.A.; Troitsky, S.V. Directional Association of TeV to PeV Astrophysical Neutrinos with Radio Blazars. Astrophys. J. 2021, 908, 157. [CrossRef]

21. Plavin, A.; Plavin, A.; Kovalev, Y.Y.; Kovalev, Y.A.; Troitsky, S. Observational Evidence for the Origin of High-energy Neutrinos in Parsec-scale Nuclei of Radio-bright Active Galaxies. Astrophys. J. 2020, 894, 101. [CrossRef]

22. Albert, A.; André, M.; Anghinolfi, M.; Anton, G.; Ardid, M.; Aubert, J.J.; Aublin, J.; Baret, B.; Basa, S.; Belhorma, B.; et al. ANTARES Search for Point Sources of Neutrinos Using Astrophysical Catalogs: A Likelihood Analysis. Astrophys. J. 2021, 911, 48. [CrossRef]

23. Illuminati, G. et al. [ANTARES Collaboration]. ANTARES search for neutrino flares from the direction of radio-bright blazars. In Proceedings of the International Cosmic Ray Conference (ICRC2021), Berlin, Germany, 12-23 July 2021; Volume 395, p. 972.

24. Aartsen, M.G.; Ackermann, M.; Adams, J.; Aguilar, J.A.; Ahlers, M.; Ahrens, M.; Altmann, D.; Andeen, K.; Anderson, T.; Ansseau, I.; et al. The IceCube Realtime Alert System. Astropart. Phys. 2017, 92, 30. [CrossRef]

25. Rodrigues, X.; Garrappa, S.; Gao, S.; Paliya, V.S.; Franckowiak, A.; Winter, W. Multiwavelength and Neutrino Emission from Blazar PKS $1502+106$. Astrophys. J. 2021, 912, 54. [CrossRef]

26. Paliya, V.S.; Böttcher, M.; Olmo-García, A.; Domínguez, A.; de Paz, A.G.; Franckowiak, A.; Garrappa, S.; Stein, R. Multifrequency Observations of the Candidate Neutrino-emitting Blazar BZB J0955+3551. Astrophys. J. 2020, 902, 29. [CrossRef]

27. Giommi, P.; Padovani, P.; Oikonomou, F.; Glauch, T.; Paiano, S.; Resconi, E. 3HSP J095507.9+355101: A flaring extreme blazar coincident in space and time with IceCube-200107A. Astron. Astrophys. 2020, 640, L4. [CrossRef]

28. Ajello, M.; Angioni, R.; Axelsson, M.; Ballet, J.; Barbiellini, G.; Bastieri, D.; Gonzalez, J.B.; Bellazzini, R.; Bissaldi, E.; Bloom, E.D.; et al. The Fourth Catalog of Active Galactic Nuclei Detected by the Fermi Large Area Telescope. Astrophys. J. 2020, 892, 10. [CrossRef]

29. Aartsen, M.G. et al. [IceCube Collaboration]. The contribution of Fermi-2LAC blazars to the diffuse TeV-PeV neutrino flux. Astrophys. J. 2017, 835, 45. [CrossRef]

30. Wang, X.Y.; Liu, R.Y.; Dai, Z.G.; Cheng, K.S. Probing the tidal disruption flares of massive black holes with high-energy neutrinos. Phys. Rev. D 2011, 84, 081301. [CrossRef]

31. Bell, E.C.; Kulkarni, S.R.; Graham, M.J.; Dekany, R.; Smith, R.M.; Riddle, R.; Masci, F.J.; Helou, G.; Prince, T.A.; Adams, S.M.; et al. The Zwicky Transient Facility: System Overview, Performance, and First Results. Publ. Astron. Soc. Pac. 2019, 131, 018002. [CrossRef]

32. Stein, R.; van Velzen, S.; Kowalski, M.; Franckowiak, A.; Gezari, S.; Miller-Jones, J.C.; Frederick, S.; Sfaradi, I.; Bietenholz, M.F.; Horesh, A.; et al. A tidal disruption event coincident with a high-energy neutrino. Nat. Astron. 2021, 908, 510-518. [CrossRef]

33. Albert, A. et al. [ANTARES Collaboration]. Search for Neutrinos from the Tidal Disruption Events AT2019dsg and AT2019fdr with the ANTARES Telescope. Astrophys. J. 2021, 920, 50. [CrossRef] 
34. Allakhverdyan, V.A. et al. [GVD-Baikal Collaboration]. Multi-messenger and real-time astrophysics with the Baikal-GVD telescope. In Proceedings of the International Cosmic Ray Conference (ICRC2021), Berlin, Germany, 12-23 July 2021; Volume 395, p. 946.

35. Stein, R. Tidal disruption event coincident with a high-energy neutrino. In Proceedings of the International Cosmic Ray Conference (ICRC2021), Berlin, Germany, 12-23 July 2021; Volume 395, p. 009.

36. Waxman, E.; Bahcall, J.N. High-energy neutrinos from cosmological gamma-ray burst fireballs. Phys. Rev. Lett. 1997, 78, 2292. [CrossRef]

37. Albert, A.; André, M.; Anghinolfi, M.; Anton, G.; Ardid, M.; Aubert, J.J.; Avgitas, T.; Baret, B.; Barrios-Martí, J.; Basa, S.; et al. Search for high-energy neutrinos from bright GRBs with ANTARES. Mon. Not. Roy. Astron. Soc. 2017, 469, 906-915. [CrossRef]

38. Aartsen, M.G. et al. [IceCube Collaboration]. An All-Sky Search for Three Flavors of Neutrinos from Gamma-Ray Bursts with the IceCube Neutrino Observatory. Astrophys. J. 2016, 824, 115. [CrossRef]

39. Bartos, I.; Veske, D.; Kowalski, M.; Marka, Z.; Marka, S. The IceCube Pie Chart: Relative Source Contributions to the Cosmic Neutrino Flux. arXiv 2021, arXiv:2105.03792.

40. Adrian-Martinez, S.; Ageron, M.; Aharonian, F.; Aiello, S.; Albert, A.; Ameli, F.; Anassontzis, E.; Andre, M.; Androulakis, G.; Anghinolfi, M.; et al. Letter of intent for KM3NeT 2.0. J. Phys. G. 2016, 43, 084001. [CrossRef]

41. Belolaptikov, I.; Dzhilkibaev, Z. [GVD-Baikal Collaboration]. Neutrino Telescope in Lake Baikal: Present and Nearest Future. In Proceedings of the International Cosmic Ray Conference (ICRC2021), Berlin, Germany, 12-23 July 2021; Volume 395, p. 002.

42. Kowalski, M. et al. [IceCube Collaboration]. IceCube: The Window to the Extreme Universe. In Proceedings of the International Cosmic Ray Conference (ICRC2021), Berlin, Germany, 12-23 July 2021; Volume 395, p. 022.

43. Aartsen, M.G.; Abbasi, R.; Ackermann, M.; Adams, J.; Aguilar, J.A.; Ahlers, M.; Ahrens, M.; Alispach, C.; Allison, P.; Amin, N.M.; et al. IceCube-Gen2: The window to the extreme Universe. J. Phys. G. 2021, 48, 060501. [CrossRef]

44. Aartsen, M.G. et al. [IceCube Collaboration]. The IceCube Neutrino Observatory-Contributions to the 36th International Cosmic Ray Conference (ICRC2019). arXiv 2019, arXiv:1907.11699.

45. Aartsen, M.G.; Abraham, K.; Ackermann, M.; Adams, J.; Aguilar, J.A.; Ahlers, M.; Ahrens, M.; Altmann, D.; Andeen, K.; Anderson, T.; et al. PINGU: A Vision for Neutrino and Particle Physics at the South Pole. J. Phys. G. 2017, 44, 054006. [CrossRef]

46. Resconi, E. The Pacific Ocean Neutrino Experiment at Ocean Networks Canada. In Proceedings of the International Cosmic Ray Conference (ICRC2021), Berlin, Germany, 12-23 July 2021; Volume 395, p. 024.

47. Abe, K. et al. [Hyper-Kamiokande Proto-Collaboration]. Hyper-Kamiokande Design Report. arXiv 2018, arXiv:1805.04163.

48. Abi, B.; Acciarri, R.; Acero, M.A.; Adamov, G.; Adams, D.; Adinolfi, M.; Ahmad, Z.; Ahmed, J.; Alion, T.; Alonso Monsalve, S.; et al. Deep Underground Neutrino Experiment (DUNE), Far Detector Technical Design Report, Volume I Introduction to DUNE. J. Instrum. 2020, 15, T08008. [CrossRef]

49. Zanin, R.; Abdalla, H.; Abe, H.; Abe, S.; Abusleme, A.; Acero, F.; Acharyya, A.; Acin Portella, V.; Ackley, K.; Adam, R.; et al. CTA-The World's largest ground-based gamma-ray observatory. In Proceedings of the International Cosmic Ray Conference (ICRC2021), Berlin, Germany, 12-23 July 2021; Volume 395, p. 005.

50. Cao, Z. Highlights of LHAASO science results. In Proceedings of the International Cosmic Ray Conference (ICRC2021), Berlin, Germany, 12-23 July 2021; Volume 395, p. 011.

51. Akutsu, T. et al. [KAGRA collaboration]. KAGRA: 2.5 generation interferometric gravitational wave detector. Nat. Astron. 2019, 3, 35-40. [CrossRef]

52. Aab, A. et al. [The Pierre Auger Collaboration]. The Pierre Auger Observatory Upgrade-Preliminary Design Report. arXiv 2016, arXiv:1604.03637.

53. The Gamma-ray Coordinates Network. Available online: https://gcn.gsfc.nasa.gov/ (accessed on 30 September 2021).

54. The Astronomer's Telegram. Available online: https://www.astronomerstelegram.org/ (accessed on 30 September 2021).

55. Reichherzer, P.; Schüssler, F.; Lefranc, V.; Yusafzai, A.; Alkan, A.K.; Ashkar, H.; Becker Tjus, J. Astro-COLIBRI-The COincidence LIBrary for Real-time Inquiry for Multimessenger Astrophysics. Astrophys. J. Supp. 2021, 256, 5. [CrossRef]

56. Adrián-Martínez, S. et al. [ANTARES, TAROT, ROTSE, Swift, Zadko Collaborations]. Optical and X-ray early follow-up of ANTARES neutrino alerts. J. Cosmol. Astropart. Phys. 2016, 2016, 062. [CrossRef]

57. Dornic, D.; Ageron, M.; Bertin, V.; Brunner, J.; Coleiro, A.; Schüssler, F.; Turpin, D.; Vallage, B. Ten years of multi-wavelength follow-up observations of ANTARES neutrino alerts. In Proceedings of the International Cosmic Ray Conference (ICRC2019), Madison, WI, USA, 24 July-1 August 2019; Volume 358, p. 871.

58. Solares, H.A.A.; Coutu, S.; Cowen, D.F.; DeLaunay, J.J.; Fox, D.B.; Keivani, A.; Mostafá, M.; Murase, K.; Oikonomou, F.; Seglar-Arroyo, M.; et al. The Astrophysical Multimessenger Observatory Network (AMON): Performance and science program. Astropart. Phys. 2020, 144, 68-76. [CrossRef]

59. Hugo, A. et al. [the AMON Group, the IceCube Collaboration, the HAWC Collaboration, the ANTARES Collaboration]. Multimessenger NuEM Alerts with AMON. In Proceedings of the International Cosmic Ray Conference (ICRC2021), Berlin, Germany, 12-23 July 2021; Volume 395, p. 958.

60. Albert, A. et al. [IceCube Collaboration]. ANTARES and IceCube Combined Search for Neutrino Point-like and Extended Sources in the Southern Sky. Astrophys. J. 2020, 892, 92. [CrossRef]

61. Albert, A. et al. [LIGO Scientific Collaboration and Virgo Collaboration]. Search for High-energy Neutrinos from Binary Neutron Star Merger GW170817 with ANTARES, IceCube, and the Pierre Auger Observatory. Astrophys. J. Lett. 2017, 850, L35. [CrossRef] 
62. Albert, A. et al. [LIGO Scientific Collaboration and Virgo Collaboration]. The Baikal-GVD neutrino telescope: First results of multimessenger studies-Contributions to the 36th International Cosmic Ray Conference (ICRC2019). arXiv 2019, arXiv:1908.05450.

63. Kimura, S.S.; Murase, K.; Bartos, I.; Ioka, K.; Heng, I.S.; Mészáros, P. Transejecta high-energy neutrino emission from binary neutron star mergers. Phys. Rev. D 2018, 98, 043020. [CrossRef]

64. González, J.P. et al. [KM3NeT Collaboration]. KM3NeT/ARCA sensitivity to transient neutrino sources. In Proceedings of the International Cosmic Ray Conference (ICRC2021), Berlin, Germany, 12-23 July 2021; Volume 395, p. 1126.

65. Oikonomou, F. High-energy neutrino emission from blazars. In Proceedings of the International Cosmic Ray Conference (ICRC2021), Berlin, Germany, 12-23 July 2021; Volume 395, p. 030.

66. Ackermann, M.; Ajello, M.; Albert, A.; Atwood, W.B.; Baldini, L.; Ballet, J.; Barbiellini, G.; Bastieri, D.; Bechtol, K.; Bellazzini, R.; et al. The spectrum of isotropic diffuse gamma-ray emission between $100 \mathrm{MeV}$ and $820 \mathrm{GeV}$. Astrophys. J. 2015, 799, 86. [CrossRef]

67. Abbasi, R. et al. [IceCube Collaboration]. The IceCube high-energy starting event sample: Description and flux characterization with 7.5 years of data. Phys. Rev. D 2021, 104, 022002. [CrossRef]

68. Fenu, F. et al. [Pierre Auger Collaboration]. The cosmic ray energy spectrum measured using the Pierre Auger Observatory. In Proceedings of the International Cosmic Ray Conference (ICRC2017), Busan, Korea, 12-20 July 2017; Volume 301, p. 486.

69. Roth, M.A.; Krumholz, M.R.; Crocker, R.M.; Celli, S. The diffuse $\gamma$-ray background is dominated by star-forming galaxies. Nature 2021, 597, 341-344. [CrossRef]

70. Aab, A. et al. [The Pierre Auger Collaboration]. An Indication of anisotropy in arrival directions of ultra-high-energy cosmic rays through comparison to the flux pattern of extragalactic gamma-ray sources. Astrophys. J. Lett. 2018, 853, L29. [CrossRef]

71. Lunardini, C.; Vance, G.S.; Emig, K.L.; Windhorst, R.A. Are starburst galaxies a common source of high energy neutrinos and cosmic rays? J. Cosmol. Astropart. Phys. 2019, 2019, 073. [CrossRef]

72. Amenomori, M. et al. [Tibet $A S_{\gamma}$ Collaboration]. First Detection of sub-PeV Diffuse Gamma Rays from the Galactic Disk: Evidence for Ubiquitous Galactic Cosmic Rays beyond PeV Energies. Phys. Rev. Lett. 2021, 126, 141101. [CrossRef]

73. Fang, K.; Murase, K. Multi-messenger Implications of Sub-PeV Diffuse Galactic Gamma-Ray Emission. Astrophys. J. 2021, 919, 93. [CrossRef]

74. Abeysekara, A.U. et al. [HAWC Collaboration]. Multiple Galactic Sources with Emission Above 56 TeV Detected by HAWC. Phys. Rev. Lett. 2020, 124, 021102,. [CrossRef]

75. Cao, Z.; Aharonian, F.A.; An, Q.; Bai, L.X.; Bai, Y.X.; Bao, Y.W.; Bastieri, D.; Bi, X.J.; Bi, Y.J.; Cai, H.; et al. Ultrahigh-energy photons up to 1.4 petaelectronvolts from $12 \gamma$-ray Galactic sources. Nature 2021, 594, 33-36. [CrossRef]

76. Dzhappuev, D.D. et al. [Carpet-3 Group]. Observation of Photons above $300 \mathrm{TeV}$ Associated with a High-energy Neutrino from the Cygnus Region. Astrophys. J. Lett. 2021, 916, L22. [CrossRef] 